\title{
Inappropriateness in biliary stenting
}

\author{
Giuseppe Chesi, Antonio Faraone, Claudio Giumelli \\ Department of Internal Medicine, Scandiano Hospital, Azienda USL Reggio Emilia, Italy
}

\begin{abstract}
Starting from a real case of a 69 -year old patient affected by cholangiocarcinoma, we intend to discuss the accuracy and appropriateness of the diagnostic and therapeutic procedures adopted. This case shows in particular that a more accurate preoperative staging could probably avoid the patient unnecessary laparotomy. According to the indications in the medical literature, this patient could possibly benefit from chemotherapy, but a chemoembolization of liver metastases was performed. However in the literature no available evidence suggests that this treatment would be beneficial in this kind of clinical picture. Eventually, when the disease was already at an advanced stage and worsened due to a necrosis of the left hepatic lobe and a cholangitic infection, a repositioning of the stent on the stent was performed, despite in the literature the life expectancy cut-off for this procedure is at least 6 months. We also discuss the communication between the physician, the patient and the family, which was probably based on overly optimistic and unrealistic expectations. This led to a number of surgical procedures, which were not certainly helpful and indeed were probably even harmful for this patient. In addition, these procedures caused unnecessary costs borne by the healthcare system. In conclusion, we advocate that discussion and self-assessment must be always promoted, so that the healthcare professionals can review the process and the outcome of their treatment as well as their behavior to understand if it could have been more appropriate to offer actual benefits to the patients in terms of better quality of life and longer life expectancy.
\end{abstract}

\section{Introduction}

In recent years, considerable progress has been made in the field of palliation of obstructive jaundice of neoplastic origin with a minimally invasive endoscopic treatment. ${ }^{1-3}$ This approach has also been complemented with percutaneous techniques, expanding their potential field of application with less invasiveness and more effective results. Groups of expert and various scientific societies have collaborated to give indications and guidance about their role. ${ }^{4}$ These techniques are in fact palliative and not curative, and this must be quite rightly their role within the therapeutic flow chart for patients with obstructive jaundice of neoplastic origin. However, in the clinical practice, these guidelines are

Correspondence: Giuseppe Chesi, UOC Medicina Interna, Ospedale C. Magati, viale Martiri della Libertà 2, 42019 Scandiano (RE), Italy.

E-mail: chesig@ausl.re.it

Key words: cholangiocarcinoma, biliary stent, appropriateness.

Received for publication: 8 May 2013.

Revision received: 29 May 2013.

Accepted for publication: 24 June 2013.

This work is licensed under a Creative Commons Attribution NonCommercial 3.0 License (CC BY-NC 3.0).

(C) Copyright G. Chesi et al., 2014

Licensee PAGEPress, Italy

Italian Journal of Medicine 2014; 8:121-123

doi:10.4081/itjm.2013.383 not always followed and often, as recently reported about chemotherapy in patients with advanced malignancies, patients and families do not receive accurate information about the effectiveness and the real purpose of these treatments. ${ }^{5}$ On the basis of this real case, we argue that not only does the inappropriateness of these treatments lead an unjustified increase in healthcare costs, but it also often has negative outcomes in terms of palliation and therefore control of symptoms.

\section{Case Report}

The patient was a 69 -year old woman who first examined in November 2011 due to an obstructive jaundice triggered by a biliary cancer diagnosed with a computerized tomography (CT) scan. Since this condition was worsening and symptomatic, for palliation purposes the patient first underwent echoendoscopy with biopsy of the hilar lesion of the liver (cholangiocarcinoma). Subsequently she underwent a successful endoscopic retrograde cholangiopancreatography and a temporary plastic stent implantation for the palliation of jaundice. At that time no data suggested an associated cholangitis. Surgery was then attempted but, because of the presence of liver metastases, was not undertaken any medical treatment. A month after surgery, the patient was hospitalized again and had a definitive palliation surgery in which the previous plastic stent was replaced with a new bare-metal stent. The patient then had an acceptable quality of life and, after five months, in June 2012, she underwent chemoembolization to treat the metastatic liver lesions mainly 
located in the left lobe of the liver. Then a febrile phase followed. This was interpreted as a consequence of the necrosis at the lesion site, which was documented in a CT scan performed the following month, at the end of July. A month later, the patient started to develop symptoms such as nausea, loss of appetite, sometimes vomiting with febrile episodes accompanied by shivering. When she came to our attention, she had neutrophil leukocytosis and a slight deterioration of renal function. A new CT scan showed a hepatic necrosis involving almost the entire left lobe. After admission to hospital, the patient had a worsening of jaundice and neutrophil leukocytosis, procalcitonin increased and various blood cultures tested positive for a gramnegative bacterium (Enterobacter cloacae). The patient was treated and improved with piperacillin/ tazobactam. She was subsequently discharged after explaining her family members that the prognosis was very severe and, given the infection started from the biliary tract where the metal stent was previously implanted, it would be high unlikely for the patient to recover fully, because the tumor was progressing and the hepatic necrosis was significant. However, after discharge, the family took the patient back to the centre where the metallic stent was implanted. The endoscopy team performed a new endoscopic (maneuver) operation to reopen partially the stent and reposition the stent infrastent. After this new surgery, high fever reappeared and a new gram bacterium producing extended-spectrum $\beta$-lactamases was isolated. The patient continued to worsen and died 20 days after this procedure.

\section{Discussion}

The case described above lends itself very well to assess the cost/benefit ratio of every individual step of this procedure and to express an appropriate opinion on the efficacy of procedures and operations suggested in the case of an advanced and inoperable tumor of the biliary tract with particular reference to the correct indications for the implantation of a biliary stent.

A thorough assessment of the various steps taken in this clinical case led to the following considerations.

\section{Initial palliative plastic stent implantation to treat worsening jaundice}

The patient was at the initial stage of the disease and presented symptoms associated with obstructive jaundice. The diagnosis and the staging of the tumor had not yet been completed and surgery was not yet completely excluded. Consequently, also in keeping with the indications of the literature and the major scientific societies, $, 2,6$ the plastic stent implantation can be considered appropriated and indicated.

\section{Major surgery}

Given the relatively young age and life expectancy of the patient, surgery could and had to be taken into account. ${ }^{6,7}$ However, given the type of tumor and its hepatic perihilar localization, staging was only performed with CT, whereas magnetic resonance or positron emission tomography ${ }^{8}$ could offer greater precision and enable physicians to rule out surgery, which then proved to be useless and potentially harmful for the patient.

\section{Placement of metallic stent for final palliation}

The patient was relatively young and partly recovered after surgery. Considering that the previously implanted plastic stent, according to the available data, would close in a short time (3-4 months), the definitive palliation surgery with metallic stent implantation was therefore an appropriate treatment. In fact, even according to the indications of the latest guidelines issued by the major scientific societies that worked on this topic, the implantation of this kind of stent is recommended, when life expectancy exceeds 6 months. ${ }^{1,2,9}$ This was reasonable at the time of the implantation, since the patient could be reasonably benefit from a combined chemotherapy treatment, which, according to the literature, might have offered a further 3-4 month survival. ${ }^{7,10,11}$ Surprisingly enough, this option was first proposed to the patient, but, 4 months after the placement of the metallic stent, a chemoembolization treatment was suggested and performed.

\section{Chemoembolization}

Chemoembolization is mainly recommended for patients with inoperable multifocal hepatocellular carcinoma. ${ }^{12}$ In this field, no conclusive data is available in the literature, and, even if some encouraging results were reported in some studies, even a recent Cochrane review ${ }^{13}$ was not in favor of this treatment. As regards cholangiocarcinoma, data is very limited, and there is no evidence of any benefit from this treatment, also considering that complications are far from negligible. ${ }^{14}$

\section{Metal stent repositioning on the previous stent}

Ten months after the inoperable Cholangiocarcinoma was diagnosed, given the cancer progression and a near complete necrosis of the entire left lobe of the liver, the prognosis appeared reasonably less than six months considering also that the one-year survival for this tumor is lower than $20 \%$. Therefore, even according to the data in the literature and the indications from various consensus papers, there was no indication for repositioning the stent. ${ }^{1,2,3,15}$ Furthermore, the presence of cholangitis was another factor to consider and should 
have discouraged from performing surgery which could spread the infection even further, since the previous stent was not removable. ${ }^{15}$

\section{Conclusions}

The case described in all its phases is a clear example of medical malpractice. On the one hand, it can be mainly ascribed to inadequate information given to the patient and her family, which generated excessive and unrealistic expectations that did not reflect what these methods could actually offer. On the other hand, it stems from excessive self-reference of the professionals involved. In fact, if no blame can be put on the correct execution and the skills of the experienced professionals who performed the procedures, the same cannot be said for the appropriateness of these procedures and the accuracy of the information provided. This process led the patient to undergo useless and ineffective quoad vitam et quoad valetudinem operations and treatments (surgery, chemoembolization), that certainly had a negative impact on her quality of life. At the same time, the cost of these procedures borne by the healthcare system were not at all negligible. Consequently, taking into account the subsequent procedures and the outcomes of patients, it would be important and useful for the skilled medical and surgical teams (along with other professionals, methodologists and employees in the departments) that often carry out these procedures, to review occasionally their work and the correctness of their statements both before and after treatment. This can certainly improve communication with patients and their relatives, their health and quality of life as well as cost containment as a result of greater appropriateness of procedures performed.

\section{Key messages}

- The placement of a biliary stent is an effective palliative operation in patients with obstructive jaundice of neoplastic origin, when life expectancy is longer than 6 months; in this case, the placement of a metallic stent was indicated.

- A plastic stent may be placed while waiting for an accurate tumor staging to be completed and a feasibility assessment of radical surgery.

- The chemotherapy regimen with gemcitabine and oxaliplatin is the only treatment that can prolong survival and improve quality of life in the presence of obstructive jaundice caused by a cholangiocarcinoma that cannot be controlled with radical surgery.

- There is no evidence, nor adequate literature available to support chemoembolization for the treatment of advanced cholangiocarcinoma.

\section{References}

1. Moss AL, Morris A, Leyden J, MacMathuna P. Malignant distal biliary obstruction: a systematic review and meta-analysis of endoscopic and surgical by-pass results. Cancer Treat Rev 2007;33:213-31.

2. Fujita N. Endoscopic management of biliary malignances. Gastrointest Endosc Clin N Am 2008;18:565-79.

3. Lee JH. Self-expandable metal stent for malignant distal biliary strictures. Gastrointest Endosc Clin N Am 2011; 21:463-80.

4. Alvaro D, Cannizzaro R, Labianca R. Cholangiocarcinoma: a position paper by the Italian Society of Gastroenterology (SIGE), the Italian Association of Hospital Gastroenterology (AIGO), the Italian Association of Medical Oncology (AIOM) and the Italian Association of Oncological Radiotherapy (AIRO). Dig Liver Dis 2010;42:831-8.

5. Weeks JC, Catalano PJ, Cronin A, et al. Patients' expectation about effects of chemotherapy for advanced cancer. N Engl J Med 2012;367:1616-25.

6. Aljiffry M, Walsh MJ, Molinari M. Review: advances in diagnosis, treatment and palliation of cholangiocarcinoma: 1990-2009. World J Gastroenterol 2009;15: 4240-62.

7. Eckel F, Brunner T, Jelic S. Biliary cancer: ESMO Clinical Practice Guidelines for diagnosis, treatment and follow up. Ann Oncol 2011;22:v40-4.

8. Sharma B, Martin A, Zerizer I. Positron emission tomography-computed tomography in liver imaging I. Semin Ultrasound CT MR 2013;34:66-80.

9. Neal CP, Thomasset SC, Bools D, et al. Combined percutaneous-endoscopic stenting of malignant biliary obstruction: results from 106 consecutive procedures and identification of factors associated with adverse outcome. Surg Endosc 2010;24:423-31.

10. Sharma A, Dwary AD, Mohanti BK, et al. Best supportive care compared with chemotherapy for unresectable gallbladder cancer: a randomized controlled study. J Clin Oncol 2010;28:4581-6.

11. Valle J, Wasan H, Palmer DH, et al. Cisplatin plus gemcitabine versus gemcitabine for biliary tract cancer. $\mathrm{N}$ Engl J Med 2010;362:1273-81.

12. Memon K, Lewandowski RJ, Kulik L, et al. Radioembolization for primary and metastatic liver cancer. Semin Radiat Oncol 2011;21:294-302.

13. Oliveri RS, Wetterslev J, Gluud C. Transarterial (chemo)embolisation for unresectable hepatocellular carcinoma (Review). Cochrane Database Syst Rev 2011; 16:CD004787.

14. Sun Z, Li G, Ai X, et al. Hepatic and biliary damage after transarterial chemoembolization for malignant hepatic tumours: incidence, diagnosis, treatment, outcome and mechanism. Crit Rev Oncol Hemat 2011;79:164-74.

15. Catalano O, De Bellis M, Sandomenico F, et al. Complication of biliary and gastrointestinal stents: MDCT of the cancer patients. AJR 2012 Am J Roentgenol 2012; 199:W187-96. 